\title{
Desenvolvendo um modelo de revisão rápida para graduação em Educação Física
}

\author{
Developing a rapid review model for undergraduate level in Physical Education
}

Crisllayne Fátima de Souza ${ }^{1}$, Verônica Volski Mattes ${ }^{1}$, Michael Pereira da Silva ${ }^{1}$, Taniya Singh Nagpal ${ }^{2}$, Danilo Fernandes da Silva ${ }^{1,2}$

${ }^{1}$ Universidade Estadual do Centro-Oeste do Paraná (Unicentro), Guarapuava/PR, Brasil

2 University of Ottawa (uOttawa), Ottawa/ON, Canadá

\section{HISTÓRICO DO ARTIGO}

Recebido: 14 dez 2019

Revisado: 26 maio 2020

Aprovado: 28 maio 2020

\section{PALAVRAS-CHAVE:}

Atenção primária à saúde; Exercício; Saúde Pública; Centros de Saúde; Gestação.

\section{KEYWORDS:}

Primary Health Care; Exercise; Public Health; Health Centers; Pregnancy.

\section{RESUMO}

OBJETIVO: Elaborar um modelo de revisão rápida que pode ser útil para trabalhos de conclusão de curso (TCC) de Educação Física, e para a aproximação entre evidências científicas e prática profissional.

MÉTODOS: São descritas as adaptações feitas para criar um método alternativo de condução de revisões rápidas para TCC's de curso de graduação.

RESULTADOS: Aluna e professor apresentaram os dados extraídos dos estudos, opinião inicial e síntese final da aluna e, finalmente, relato crítico de membros da banca e pesquisadora com experiência em tradução de conhecimento (Knowledge Translation, KT) sobre a estratégia desenvolvida froam incluídos.

CONCLUSÃO: Espera-se que esse relato propicie discussão continuada sobre o processo de orientação de TCC's na Educação Física, neste caso com ênfase em Atividade Física e Saúde e formas de se fazer a evidência científica se aproximar da prática, através de ações direcionadas aos futuros profissionais.

\section{ABSTRACT}

OBJECTIVE: To elaborate on a rapid review model that can be useful for undergraduate thesis projects (TCC) in Physical Education courses, and for approximate science and professional practice.

METHODS: We described the adaptations performed in order to come up with an alternative method to develop rapid reviews in fourth year projects at the undergraduate level.

RESULTS: A student and professor presented extracted data from the studies, the student provided initial opinion and final synthesis and, finally, critical appraisal of the committee and researcher with expertise in knowledge translation about the developed method were included.

CONCLUSION: We expect that this report will promote discussion on the topic about the supervision of fourth year research projects in Physical Education, with emphasis on Physical Activity and Health and offers a way to bridge the gap between scientific evidence and practices through actions directed at to future professionals. 


\section{INTRODUÇÃo}

O trabalho de conclusão de curso (TCC) é uma etapa muito aguardada pelos alunos e pelos departamentos de cursos com esta avaliação, normalmente ocorrendo no último ano da graduação (DEDUF, 2014). O TCC no curso de Educação Física (bacharelado) costuma ter um papel de conciliar ferramentas técnico-científicas na elaboração de um estudo seguindo normas acadêmicas, em que aluno e orientador concordam em um tópico de investigação e determinam, primeiramente, um projeto, para na sequência executá-lo e, finalmente, relatálo no tão aguardado dia da defesa. Para os alunos, é uma oportunidade de selecionar um tema dentro de seu campo de interesse, que no caso deste curso de graduação, pode variar em diversas áreas, como treinamento desportivo, biomecânica, dança, lazer, comportamento motor, atividade física e saúde, entre outros exemplos.

Em geral, no Departamento de Educação Física da Universidade Estadual do Centro-Oeste - Unicentro (DEDUF, 2014), os alunos costumam desenvolver estudos de campo para poderem ter contato com uma população que tem interesse em futuramente trabalhar, realizar avaliações que poderão ser comuns na sua rotina de trabalho ou os ensinarão sobre respostas físicas, fisiológicas, cognitivas, comportamentais que os interessam. Além disso, este tipo de estudo vai requerer subsequentemente uma análise quantitativa ou qualitativa das informações obtidas. Caso não optem por um estudo de campo, os acadêmicos acabam realizando um estudo de revisão de literatura.

Em muitos casos parece que a escolha pelo estudo de revisão é uma segunda opção e, infelizmente, está atrelada à falta de tempo para concluir o estudo de campo, impossibilidade de realizar as avaliações desejadas pelo aluno, ou casos em que algumas dificuldades ao longo do processo de orientação geram uma troca de orientador. Dentre os tipos de revisão, é mais comum que orientadores e alunos realizem as revisões narrativas, que são mais amplas no processo de busca e seleção da literatura, não incluem a descrição de um método definido e tornam-se exageradamente empíricas, inconclusivas e pautadas demasiadamente na análise crítica e pessoal do(s) autor(es) (ROTHER, 2007; GOMES; CAMINHA, 2014). Considerando esses aspectos, os resultados obtidos com estudos de revisão narrativa em TCC acabam ficando limitados, resultando em pouca aplicação acadêmica (e.g., publicação, divulgação aos pares) ou profissional (e.g., releitura por parte de profissionais e aplicação no campo de atuação - knowledge translation - KT) (WHO, 2006).

Analisando o perfil das revisões publicadas nos principais periódicos científicos da Educação Física, é notável que as revisões narrativas têm perdido espaço e as revisões sistemáticas são preferíveis. Isso é natural, considerando a posição das revisões sistemáticas na pirâmide de nível de evidência e seu potencial de contribuição científica (CHALMERS, 2005; LAVIS et al., 2009). No entanto, esse processo demanda tempo, uma equipe de 3 ou mais membros e experiência no processo. Como possuem procedimentos e análises complexas, já foi notado que as revisões sistemáticas podem desencorajar profissionais e formadores de políticas públicas a usarem informação científica na sua tomada de decisões na prática, já que precisam de tempo e capacidade de sintetizar as informações mais relevantes (OLIVER et al., 2014).

Pensando nisso, uma série de métodos para as chamadas "revisões rápidas", que adaptam e facilitam os procedimentos de revisões sistemáticas para obtenção de evidências científicas tem sido desenvolvida e aplicada na prática, para auxiliar profissionais e tomadores de decisões (e.g., políticas públicas) (POLISENA et al., 2015; KHANGURA et al., 2014). Uma revisão sistemática sobre métodos de realização de revisões rápidas sugeriu que há evidências que este tipo de revisão pode melhorar a acessibilidade e clareza das evidências científicas (HABY et al., 2016). No entanto, até onde se tem conhecimento, não há relatos de procedimentos para realização de revisões rápidas e potenciais resultados em TCC's no nível de graduação (bacharelado em Educação Física). Esse procedimento pode contribuir com a maior qualidade de estudos de revisão de alunos graduandos, bem como, favorecer maior aplicação das evidências científicas levantadas na atuação profissional, o que é um papel importante dos cursos de Educação Física (BRANDT et al., 2020).

Diante disso, o objetivo desse trabalho foi elaborar um modelo de revisão rápida que pode ser útil para trabalhos de conclusão de curso (TCC) de Educação Física, e para a aproximação entre evidências científicas e prática profissional. Para tanto, foi relatada uma experiência acadêmica entre orientador e aluna de TCC na elaboração dos métodos e execução de uma revisão rápida, focada na caracterização de programas de exercícios físicos para gestantes, visando favorecer a atuação desta aluna após formada.

\section{MÉTOdOS}

\section{Apresentando a proposta dentro da disciplina de TCC}

A Disciplina de "Trabalho de Conclusão de Curso" no departamento de Educação Física da Unicentro é realizada no último (quarto) ano e é precedida (terceiro ano) da disciplina de "Metodologia da Pesquisa em Educação Física", que almeja a elaboração do projeto para realização do TCC no ano seguinte.

$\mathrm{Na}$ disciplina do terceiro ano, o professor responsável pela mesma orienta os alunos a buscarem um professor do departamento para supervisioná-los. A aluna (CFS) em questão buscou o professor orientador (DFdS) já com a intenção de realizar um estudo de revisão de literatura que focasse na caracterização de exercícios físicos aquáticos em gestantes. Seu interesse era guiado pela possibilidade de atuação profissional com essa população, sobretudo no ambiente aquático. No entanto, se sentia insegura sobre as características do programa de exercícios físicos e sobre como desenvolver a prescrição dos mesmos em meio aquático, por se tratar de uma população especial e por receio de gerar respostas negativas para a gestante e, principalmente, para o feto.

Considerando as leituras prévias do professor sobre o potencial das revisões rápidas, os principais procedimentos para elaboração e orientação de revisões rápidas para profissionais da saúde e tomadores de decisões foram levantados. A revisão sistemática sobre métodos de revisão rápida de Haby et al. (2016) foi o ancoradouro para a adaptação de um procedimento de revisão rápida em nível de TCC. 


\section{Experiência 1: Sugestões extraídas de Haby et al. (2016)}

A ideia deste passo era embasar os procedimentos para a realização da revisão rápida. Uma referência de destaque usada por Haby et al. (2016) foi a chamada da Organização Mundial da Saúde (OMS) para estimular mecanismos de transferência de conhecimento que dão suporte a políticas públicas em saúde e práticas de saúde baseadas em evidências (WHO, 2005). Estes mecanismos fazem parte de ações de KT, termo internacionalmente reconhecido e definido como síntese, troca e aplicação de conhecimentos pelas partes interessadas (e.g., profissionais da saúde, tomadores de decisões públicas) para acelerar os benefícios de inovação local e global no fortalecimento de sistemas de saúde, prática de profissionais e saúde da população envolvida nas intervenções (WHO, 2006). Como as principais barreiras para uso de evidências científicas pelos profissionais em sua prática estão atreladas à dificuldade de acessar evidências científicas e a falta de resultados de pesquisas oportunos e relevantes (OLIVER et al., 2014), o próprio estudo de Haby et al. (2016) reforça a utilização de revisões rápidas para minimizar essa barreira.

Basicamente, as principais sugestões e adaptações na condução de revisões rápidas são extraídas dos estudos de revisão sistemática tradicionais. A Tabela 1 sintetiza os exemplos de modificações adaptados de Haby et al. (2016).

Tabela 1. Síntese de exemplos de modificações das revisões sistemáticas tradicionais para as revisões rápidas.

\begin{tabular}{|c|c|}
\hline Ordem & Exemplo de modificação* \\
\hline 1 & Pergunta de pesquisa mais delineada (escopo reduzido). \\
\hline 2 & $\begin{array}{l}\text { Redução dos tipos de estudos incluídos (foco em tipos de maior qua- } \\
\text { lidade). }\end{array}$ \\
\hline 3 & Artigos apenas na língua inglesa (língua "oficial" da ciência). \\
\hline 4 & Reduzir a faixa de tempo da busca. \\
\hline 5 & $\begin{array}{l}\text { Exclusão da literatura cinzenta (publicações não indexadas, anais de } \\
\text { eventos). }\end{array}$ \\
\hline 6 & $\begin{array}{l}\text { Facilitação do uso de ferramentas de busca (e.g., base mais acessível e } \\
\text { simplificação da busca). }\end{array}$ \\
\hline 7 & $\begin{array}{l}\text { Uso de apenas um revisor para seleção de estudos e/ou extração de } \\
\text { dados }\end{array}$ \\
\hline
\end{tabular}

Experiência 2: Processo de orientação da aluna e condução da revisão rápida

A experiência 2 é relatada seguindo a ordem dos exemplos de modificação trazidos na Tabela 1.

Exemplo 1: A grande dúvida da aluna era: "Se trabalhar em um ambiente que ofereça atividades aquáticas, e se tiver um grupo de gestantes, como devo proceder na prescrição dos exercícios físicos aquáticos para essa população?" Pensando nisso, delineou-se a pergunta de pesquisa da seguinte maneira: "quais são as características dos exercícios físicos em ambiente aquático para gestantes descritos na literatura científica?". Para reduzir o escopo de "características", focamos nos princípios FITT (frequência, intensidade, tipo e tempo) do American College of Sports Medicine (ACSM, 2018) e decidiu-se adaptá-lo na fase de extração de dados (explicada na experiência 4 mais a frente).

Exemplo 2: A aluna foi orientada a realizar a busca focada em estudos de intervenção, preferencialmente, randomizados e controlados.

Exemplo 3: O uso restrito de artigos em língua inglesa foi adotado seguindo a recomendação.

Exemplo 4: Como o tópico pesquisado era relativamente específico e não se tinha conhecimento prévio de outro estudo de revisão buscando objetivos semelhantes, optou-se por não restringir a faixa de tempo da busca.

Exemplo 5: Não foi adotada nenhuma base ou esquema de busca para identificação de literatura cinzenta, conforme recomendado.

Exemplo 6: Considerando o enfoque da pergunta do estudo ligado à saúde, optou-se por restringir as buscas ao PubMed, tendo em vista a facilidade de acesso e aplicação de buscas simplificadas. No que tange à busca, adaptou-se a estratégia PICO (População, Intervenção, Comparador, Outcome [desfecho]) (SANTOS et al., 2007), transformando-a em "PI" (população e intervenção) para simplificar o processo. Mais detalhes são relatados na experiência 3.

Exemplo 7: Após a execução da busca, a aluna foi responsável pela seleção dos estudos e extração dos dados.

\section{Experiência 3: 0 processo de busca do PubMed: estratégia "PI"}

A estratégia PICO foi simplificada nessa experiência. Considerando que a pergunta de pesquisa foi mais restrita, não seria necessário focar em comparadores " $C$ " ou desfechos específicos "O". Além do mais, o uso de menos letras da estratégia PICO deixa a mesma mais sensível ao que de fato estava se procurando, o que seria benéfico para o objetivo.

Para facilitar ainda mais o processo, a busca foi feita no PubMed sem o uso da ferramenta de busca avançada. Nesta busca, a aluna e professor optaram por usar os seguintes termos "pregnant women" ou "pregnancy" para "P" (população) e "water exercise" ou "aquatic exercise" para "I".

Um termo da "população" foi combinado com um termo da "intervenção" e os dois foram separados pelo termo booleano "AND" para verificar qual combinação resultaria na maior quantidade de estudos. A busca registrou mais estudos ao ser feita da seguinte forma: "pregnancy AND water exercise" = total de 257 resultados em 04/04/2019 e atualizada em 08/10/2019 totalizando 262 resultados.

Dentre os 262 estudos, 28 foram incluídas pela aluna após análise por título e resumo e, finalmente, 17 passaram pela análise de texto completo e foram para extração de dados.

\section{RESULTADOS}

Experiência 4: 0 processo de extração de dados: estratégia "FIT"

O ACSM (2018) apresenta recomendações para prescrição de exercícios físicos para saúde e usa o princípio FITT (frequência semanal; intensidade do exercício; tipo de exercício; tempo que dura uma sessão) para caracterização das variáveis de prescrição. Portanto, para caracterizar os exercícios físicos aquáticos dos estudos selecionados, a aluna foi orientada a 
Tabela 2. Caracterização da aplicação dos exercícios aquáticos para gestantes de acordo com os princípios FITT e os principais desfechos avaliados.

\begin{tabular}{|c|c|c|c|c|c|c|c|}
\hline Autor (ano) & Freq. & Int. & $\begin{array}{c}t \\
(\min )\end{array}$ & Tipo & Duração & $\begin{array}{l}\text { Temp. } \\
\left({ }^{\circ} \mathrm{C}\right)\end{array}$ & Desfecho Principal \\
\hline $\begin{array}{l}\text { Rodríguez-Blanque et al. } \\
\text { (2019) }\end{array}$ & $3 x /$ sem & $\begin{array}{l}\text { Moderada } \\
\text { (12-14 Escala de Borg) }\end{array}$ & 60 & Aeróbio + Resistido & $\begin{array}{c}17 \\
\text { semanas }\end{array}$ & --- & $>$ Períneo intacto \\
\hline $\begin{array}{l}\text { Rodríguez-Blanque et al. } \\
\text { (2019) }\end{array}$ & $3 x /$ sem & --- & 60 & Aeróbio + Resistido & $\begin{array}{c}17 \\
\text { semanas }\end{array}$ & --- & < Duração do parto \\
\hline Bacchi et al. (2017) & $3 x /$ sem & $\begin{array}{l}\text { Leve a moderada } \\
\text { (10-14 Escala de Borg) }\end{array}$ & $55-60$ & $\begin{array}{l}\text { Aeróbio e dança + resistidos + natação, } \\
\text { exceto borboleta (Bolas de borracha, } \\
\text { pranchas, pull-boys, macarrões, brace- } \\
\text { letes e anéis de borracha) }\end{array}$ & $\begin{array}{l}25,8 \pm 3,3 \\
\text { semanas }\end{array}$ & $28,5-29$ & GPG mais adequado \\
\hline Backhausen et al. (2017) & $2 x /$ sem & $\begin{array}{l}\text { Moderada } \\
\text { (11-15 Escala de Borg) }\end{array}$ & $55-60$ & $\begin{array}{l}\text { Seis exercícios resistidos ( } 2 \text { halteres, } \\
\text { cinta e kickboard) } 2 \times 15-20 \text { rep }\end{array}$ & $\begin{array}{c}12 \\
\text { semanas }\end{array}$ & --- & $<$ dor lombar \\
\hline Barakat et al. (2017) & $3 x /$ sem & $\begin{array}{l}\text { Moderada } \\
\text { (12-14 Escala de Borg) }\end{array}$ & $55-60$ & $\begin{array}{l}\text { Aeróbio e dança + resistidos + natação, } \\
\text { exceto borboleta (Bolas de borracha, } \\
\text { pranchas, pull-boys, macarrões, brace- } \\
\text { letes e anéis de borracha) }\end{array}$ & $\begin{array}{c}28-31 \\
\text { semanas }\end{array}$ & $28,5-29$ & $\begin{array}{l}\text { Previne diabetes gesta- } \\
\text { cional }\end{array}$ \\
\hline Carpenter et al. (2015) & $1 x /$ sem & Leve a moderada & 40 & $\begin{array}{l}\text { Aeróbio (marcha e trote) com variados } \\
\text { movimentos de braço }\end{array}$ & $\begin{array}{l}\sim 17-20 \\
\text { semanas }\end{array}$ & --- & $\begin{array}{c}\text { Melhor ejeção ventricular } \\
\text { e menor PA }\end{array}$ \\
\hline Cordeiro et al. (2015) & $3 x /$ sem & $\begin{array}{l}\text { Moderada } \\
\text { (12-14 Escala de Borg) } \\
\text { e }<60 \% \text { FCR }\end{array}$ & $50-60$ & $\begin{array}{l}\text { Aeróbio (natação) + Resistidos (propul- } \\
\text { são) }\end{array}$ & $\begin{array}{l}\sim 25-30 \\
\text { semanas }\end{array}$ & $28,5-29$ & $\begin{array}{l}\text { Previne diabetes gesta- } \\
\text { cional }\end{array}$ \\
\hline Backhausen et al. (2014) & $2 x /$ sem & $\begin{array}{l}\text { Moderada } \\
\text { (11-15 Escala de Borg) }\end{array}$ & 45 & $\begin{array}{l}\text { Seis exercícios resistidos ( } 2 \text { halteres, } \\
\text { cinta e kickboard) } 2 \times 15 \text {-20rep }\end{array}$ & $\begin{array}{c}12 \\
\text { semanas }\end{array}$ & --- & $\begin{array}{l}>\text { Bem-estar físico e } \\
\text { mental }\end{array}$ \\
\hline Vallim et al. (2011) & $3 x /$ sem & $\begin{array}{l}\text { Moderada } \\
\text { (55-65\% FCmax) }\end{array}$ & 50 & Aeróbio & $\begin{array}{c}\sim 16 \\
\text { semanas }\end{array}$ & $28-30$ & $>$ Qualidade de vida \\
\hline Cavalcante et al. (2009) & $3 x /$ sem & $\begin{array}{l}\text { Moderada } \\
(\sim 70 \% \text { FCmax })\end{array}$ & 50 & Aeróbio & $\begin{array}{c}\sim 16 \\
\text { semanas }\end{array}$ & $28-30$ & Foi seguro \\
\hline Baciuk et al. (2008) & $3 x /$ sem & $\begin{array}{l}\text { Moderada } \\
(\sim 70 \% \text { FCmax })\end{array}$ & 50 & Aeróbio & $\begin{array}{c}\sim 16 \\
\text { semanas }\end{array}$ & $28-30$ & $<$ Analgesia no parto \\
\hline Granath et al. (2006) & $1 x /$ sem & --- & 60 & $\begin{array}{l}\text { Aeróbio (Principal) + Resistido + Flexi- } \\
\text { bilidade }\end{array}$ & $\begin{array}{l}\sim 25-28 \\
\text { semanas }\end{array}$ & --- & $<$ dor lombar \\
\hline Kihlstrand et al. (1999) & $1 x /$ sem & --- & 60 & $\begin{array}{l}30 \text { minutos de exercícios }+30 \text { minutos } \\
\text { de relaxamento }\end{array}$ & $\begin{array}{l}17-20 \\
\text { sessões }\end{array}$ & $32-34$ & $\begin{array}{l}\text { Sem risco de infecção } \\
\text { urinária ou vaginal. < Dor } \\
\text { lombar }\end{array}$ \\
\hline \multicolumn{8}{|l|}{ Desfecho } \\
\hline $\begin{array}{l}\text { Opinião da aluna } \\
\text { antes do TCC }\end{array}$ & $2 x /$ sem & Leve a moderada & 45 & Hidroginástica & $\begin{array}{l}\text { Trimestre } \\
2 \text { e } 3\end{array}$ & --- & --- \\
\hline $\begin{array}{l}\text { Recomendação da } \\
\text { aluna após o TCC }\end{array}$ & $3 x /$ sem & $\begin{array}{l}\text { Moderada } \\
(12-14) \text { na escala de } \\
\text { Borg }\end{array}$ & $45-60$ & $\begin{array}{l}\text { Exercícios aeróbios (caminhada/trote; } \\
\text { natação [exceto borboleta]; danças, } \\
\text { hidroginástica) + resistidos (equipa- } \\
\text { mentos aquáticos, foco em força de } \\
\text { resistência) }\end{array}$ & $\begin{array}{l}\text { Desde a } \\
\text { avaliação } \\
\text { pré-natal até } \\
\text { o final da } \\
\text { gestação }\end{array}$ & $28-30$ & $* *$ \\
\hline
\end{tabular}

**Resultados/benefícios seguem os achados nos estudos incluídos na revisão. FCmax = Frequência Cardíaca Máxima; FCR = Frequência Cardíaca e Reserva; GPG = Ganho de Peso Gestacional; PA = Pressão arterial; PN = Peso ao Nascer; Temp. = Temperatura.

montar uma Tabela que contivesse essas 4 informações da prescrição para cada estudo de intervenção incluído. Esse procedimento é descrito na Tabela 2, assim como a opinião inicial e síntese final feita pela aluna.

\section{Experiência 5: Apresentação para banca e discussão com especialista em KT}

O professor orientador e a aluna contaram com a análise crítica de dois subgrupos na realização desta experiência inovadora: professores do Departamento de Educação Física da Unicentro, membros da banca da aluna e uma pesquisadora canadense com experiência em KT, exercício físico e gestação.

Em suma, os professores (VVM e MPdS) consideraram que o método adaptado pode ser aplicado com seus alunos de TCC em seus temas de pesquisa no futuro. Segundo eles, o método coloca o aluno em destaque na obtenção das informações, otimiza o tempo e realiza a revisão seguindo uma sistematização que facilita sua reprodução. Eles destacaram o entusiasmo da aluna na defesa, sinceridade em apresentar suas dúvidas antes do estudo e aprendizagem da busca por evidências para ajudála na prática profissional. De fato, a aluna já está em contato com academia de exercícios aquáticos da cidade para iniciar os trabalhos com a população estudada. A segurança da aluna para 
trabalhar com tal população, segundo eles, é uma forma de ratificar a capacidade desse modelo em capacitar o acadêmico na temática escolhida no TCC.

A especialista em KT (TSN) convidada para fazer parte desta experiência tinha como papel analisar criticamente se a estratégia montada pela aluna e professor e seus resultados poderiam servir como ferramenta de KT. A pesquisadora fez parte do time de pesquisadores canadenses que recentemente publicaram o "2019 Canadian Guideline for Physical Activity throughout Pregnancy", reconhecido hoje como o principal guia de recomendação de exercícios para gestantes e que tem como pontos fortes o embasamento em várias revisões sistemáticas, opinião de especialistas, consulta com usuários e consideração sobre aplicabilidade, aceitação, custos e equidade (MOTTOLA et al., 2018). Segundo ela, uma efetiva ação de KT visa potencializar a aplicação dos resultados da pesquisa em ambiente apropriado e requer colaboração com os "usuários" de conhecimento. Os "Usuários" seriam aqueles cuja mensagem principal do estudo é mais relevante e aqueles engajados na aplicação prática e compartilhamento desses achados (JULL; GILES; GRAHAM, 2017).

A especialista ressaltou que o método adaptado das revisões rápidas tem potencial para ser aplicado como iniciativa de KT, pois a informação é sumarizada de forma sistemática. Este tipo de revisão sugere informações práticas à profissionais e ajuda a identificar lacunas na literatura. Como resultado, as revisões rápidas informam concisamente o que pode ser implementado por profissionais. Também foi ressaltado que, como esse modelo foi adaptado pensando nas necessidades e características de alunos de graduação, os métodos de revisões rápidas ajudam os estudantes a avaliar mais criticamente a literatura científica e formas de aplicar o conhecimento na prática futura. No geral, alunos aprenderão como revisar a literatura sem consumir muito tempo e produzirão conclusões focadas em sua atuação profissional.

Sua recomendação como especialista em KT para próximos passos inclui: i) a publicação da experiência em periódico científico; ii) divulgação da publicação e experiência relatada nas mídias locais (dentro e fora da universidade); iii) estímulo da repetição do método adaptado pelo próprio professor e pares da área; iv) divulgação da estratégia em cursos de especialização profissional (e.g., especializações lato sensu e residência multiprofissional); v) visitas e colaborações com academias de exercícios aquáticos locais para debater sobre os achados e compartilhar os conhecimentos adquiridos.

\section{CONCLUSÃO}

Espera-se que o relato dessas vivências adicione valor às discussões acadêmicas sobre a formação de profissionais de EF, papel dos TCC's, sobretudo trabalhos do tipo revisão de literatura, e seu potencial uso como uma atividade de KT e síntese de evidências científicas para guiarem decisões práticas, fator de grande importância para boas práticas em saúde, segundo a Organização Mundial de Saúde (OMS).

Através das experiências e interações orientador-orientanda foi possível: i) adaptar um método de revisão rápida seguindo sugestões da literatura; ii) propiciar à aluna informações que minimizassem sua insegurança e as dúvidas sobre a base de evidências científicas para prescrever exercícios aquáticos para gestantes. Segundo seu relato pessoal na defesa, este ponto foi atingido de forma muito satisfatória. Ainda assim, a aluna destacou que somar a realização de seu estudo com a experiência prática futura será crucial para seu desenvolvimento profissional na área.

Futuros passos envolvem testar esta metodologia em um número maior de alunos, verificar sua aplicação em outros temas/áreas da Atividade Física relacionada à Saúde e Educação Física, e verificar o impacto destas experiências na atuação de profissionais de Educação Física que fazem uso destas sínteses de evidências em sua prática.

\section{REFERÊNCIAS}

ACSM. American College of Sports Medicine. Diretrizes do ACSM para os testes de esforço e sua prescrição. Rio de Janeiro: Guanabara Koogan, 2018.

BACCHI, M.; MOTTOLA, M. F.; PERALES, M.; REFOYO, I.; BARAKAT, R. Aquatic activities during pregnancy prevent excessive maternal weight gain and preserve birth weight: A randomized clinical trial. American Journal of Health Promotion, Thousand Oaks, v. 32, n. 3, p. 729-35, 2018.

BACIUK, E. P.; PEREIRA, R. I.; CECATTI, J. G.; BRAGA, A. F.; CAVALCANTE, S. R. Water aerobics in pregnancy: cardiovascular response, labor and neonatal outcomes. Reproductive Health, London, v. 5, n. 1, p. 10, 2008.

BACKHAUSEN, M. G.; KATBALLE, M.; HANSSON, H.; TABOR, A.; DAMM, P. HEGAARD, $H$. K. A standardised individual unsupervised water exercise intervention for healthy pregnant women. A qualitative feasibility study. Sexual and Reproductive HealthCare, Stockholm, v. 5, n. 4, p. 176-81, 2014.

BACKHAUSEN, M. G.; TABOR, A.; ALBERT, H.; ROSTH $\varnothing$ J, S.; DAMM, P. HEGAARD, $\mathrm{H}$. K. The effects of an unsupervised water exercise program on low back pain and sick leave among healthy pregnant women - a randomised controlled trial. PloS One, San Francisco, v. 12, n. 9, p. e0182114, 2017.

BARAKAT, R.; PERALES, M.; CORDERO, Y.; BACCHI, M.; MOTTOLA, M.F. Influence of land or water exercise in pregnancy on outcomes: a crosssectional study. Medicine and Science in Sports and Exercise, Indianapolis, v. 49 , n. 7, p. 1397-403, 2017.

BRANDT, R.; MADUREIRA, A. S.; HOBOLD, E. Projetos de extensão fazendo a diferença na formação do profissional de educação física na Unioeste. Caderno de Educação Física e Esporte, Marechal Cândido Rondon, v. 18, n. 1, p. 113-7, 2020

CARPENTER, R. E.; EMERY, S. J.; UZUN, O.; D'SILVA, L. A.; LEWIS, M. J. Influence of antenatal physical exercise on haemodynamics in pregnant women: a flexible randomisation approach. BMC Pregnancy Childbirth, London, v. 15, n. 1, p. 186, 2015

CAVALCANTE, S. R.; CECATTI, J. G.; PEREIRA, R. I.; BACIUK, E. P.; BERNARDO, A L.; SILVEIRA, C. Water aerobics II: maternal body composition and perinatal outcomes after a program for low risk pregnant women. Reproductive Health, London, v. 6, p. 1, 2009.

CHALMERS, I. If evidence-informed policy works in practice, does it matter if it doesn't work in theory? Evidence \& policy: a journal of research. Debate and Practice, Bristol, v. 1, n. 2, p. 227-42, 2005.

CORDERO RODRIGUEZ, Y; MOTTOLA, M. F.; VARGAS, J.; BLANCO, M. BARAKAT CARBALLO, $R$. O Exercise in associated with a reduction in gestational diabetes mellitus. Medicine and Science in Sports and Exercise, Indianapolis, v. 47, n. 7, p. 1328-33, 2015.

DEDUF. Departamento de Educação Física. Projeto Pedagógico do curso de Educação Física de Guarapuava. Universidade Estadual do Centro-Oeste, Guarapuava, PR, 2014.

GOMES, I. S.; CAMINHA I O G Guia para estudos de revisão sistemática: uma opção metodológica para as ciências do movimento humano. Movimento, Porto Alegre, v. 20, n. 1, p. 395-411, 2014

GRANATH, A. B.; HELLGREN, M. S.; GUNNARSSON, R. K. Water aerobics reduces sick leave due to low back pain during pregnancy. Journal of Obstetrics and Gynecology Neonatal Nursing, Washington, v. 35, n. 4, p. 465-71, 2006.

HABY, M. M.; CHAPMAN, E.; CLARK, R.; BARRETO, J.; REVEIZ, L.; LAVIS, J. N. What are the best methodologies for rapid reviews of the research evidence for evidence-informed decision making in health policy and practice: a rapid review. Health Research Policy and Systems, London, v. 14, n. 1, p. 83, 2016. 
JULL, J.; GILES, A.; GRAHAM, I. D. Community-based participatory research and integrated knowledge translation: advancing the co-creation of knowledge. Implementation Science, London, v. 12, n. 1, p. 150, 2017.

KHANGURA, S.; POLISENA, J.; CLIFFORD, T. J.; FARRAH, K.; KAMEL, C. Rapid review: An emerging approach to evidence synthesis in health technology assessment. International Journal of Technology Assessment in Health Care, Cambridge, v. 30, p. 20-7, 2014

KIHLSTRAND, M.; STENMAN, B.; NILSSON, S.; AXELSSON, O. Watergymnastics reduced the intensity of back/low back pain in pregnant women. Acta Obstetricia et Gynecologica Scandinavica, London, v. 78, n. 3, p. 180-5, 1999.

LAVIS, J. N.; PERMANAND, G.; OXMAN, A. D.; LEWIN, S.; FRETHEIM A. SUPPORT Tools for evidence-informed health Policymaking (STP) 13: Preparing and using policy briefs to support evidence-informed policymaking. Health Research Policy and Systems, London, v. 7 (Suppl 1) p. S1, 2009.

MOTTOLA, M. F.; DAVENPORT, M. H.; RUCHAT, S. M.; DAVIES, G. A.; POITRAS, V. J.; ... ; ZEHR, L. 2019 Canadian guideline for physical activity throughout pregnancy. British Journal of Sports Medicine, London, v. 52, n. 21, p. 133946, 2018.

OLIVER, K.; INNVAR, S.; LORENC, T.; WOODMAN, J.; THOMAS, J. A systematic review of barriers to and facilitators of the use of evidence by policymakers. BMC Health Services Research, London, v. 14, p. 2, 2014.

POLISENA, J.; GARRITY, C.; KAMEL, C.; STEVENS, A.; ABOU-SETTA, A. M. Rapid review programs to support health care and policy decision making: a descriptive analysis of processes and methods. Systematic Reviews, London, v. 4, p. 26, 2015

RODRIGUEZ BLANQUE, R ; SÁNCHEZ GARCÍA J C.; SÁNCHEZ LÓPEZ, A. M. MUR VILLAR, N.; AGUILAR CORDERO, M. J. The influence of physical activity in water on sleep quality in pregnant women: A randomised trial. Women Birth, Camberra, v. 31, n. 1, p. e51-e58, 2017.

RODRÍGUEZ BLANQUE, R · SANCHEZ GARCIA J. C. SANCHEZ LOPEZ, A. M. EXPÓSITO RUIZ M. AGUILAR CORDERO, M.J. Randomized Clinical Trial of an Aquatic Physical Exercise Program During Pregnancy. Journal of Obstetrics and Gynecology Neonatal Nursing, Washington, v. 48, n. 3, p. 321-31, 2019.

RODRÍGUEZ BLANQUE, R.; SÁNCHEZ GARCÍA, J. C.; SÁNCHEZ LÓPEZ, A. M. AGUILAR CORDERO, M. J. Physical activity during pregnancy and its influence on delivery time: a randomized clinical trial. PeerJ, Corte Madera, v. 7, p. e6370, 2019.

ROTHER, E. T. Revisão Sistemática X Revisão Narrativa. Acta Paulista de Enfermagem, São Paulo, v. 20, n. 2, p. 5-6, 2007.

SANTOS, C. M. C.; PIMENTA, C. A. M.; NOBRE, M. R. C. The PICO strategy for research question construction and evidence search. Revista LatinoAmericana de Enfermagem, Ribeirão Preto, v. 15, n. 3, p. 508-11, 2007.

SMITH, S. A.; MICHEL, Y. A pilot study on the effects of aquatic exercises on discomforts of pregnancy. Journal of Obstetrics and Gynecology Neonatal Nursing, Washington, v. 35, n. 3, p. 315-323, 2006.

VALLIM, A. L.; OSIS, M. J.; CECATTI, J. G.; BACIUK, É. P.; SILVEIRA, C. CAVALCANTE, S. R. Water exercises and quality of life during pregnancy. Reproductive Health, London, v. 8, n. 1, p. 14, 2011.

WHO. World Health Organization. Bridging the "Know-Do" Gap: Meeting on Knowledge Translation in Global Health, 10-12 October 2005. 2006 Contract No. WHO/EIP/KMS/2006.2. Geneva: WHO; 2006.

WHO. World Health Organization. World Health Assembly. Resolution on Health Research. 2005. Disponível em: <http://www.who.int/rpc/ meetings/58th WHA resolution.pdf>. Accessedo em: 17 de novembro de 2018.

\section{AGRADECIMENTOS}

Os autores agradecem ao Departamento de Educação Física da Universidade do Centro-Oeste do Paraná (Unicentro) pelo apoio na execução do projeto.

\section{CONFLITO DE INTERESSE}

Os autores do estudo declaram não haver conflito de interesses.

\section{ORCID E E-MAIL DOS AUTORES}

Crisllayne Fátima de Souza ORCID: 0000-0002-1533-4244. E-mail: crisllaynem@hotmail.com Verônica Volski Mattes ORCID: 0000-0002-9599-6618. E-mail: vero_edf@hotmail.com Michael Pereira Da Silva ORCID: 0000-0002-7021-9847. E-mail: prof.mpsilva@outlook.com

Taniya Singh Nagpal ORCID: 0000-0003-1928-1861.

E-mail: tnagpal@uottawa.ca

Danilo Fernandes da Silva (Autor Correspondente) ORCID: 0000-0002-4170-1079.

E-mail: danilofernandesdasilva@hotmail.com

\section{FINANCIAMENTO}

Este estudo não teve nenhum tipo de apoio financeiro. 